\title{
Discriminação entre vozes adaptadas, levemente soprosas e tensas: diferenças entre os dois primeiros harmônicos
}

\section{Distinction between adapted, slightly breathy and tense voices: differences between the first two harmonics}

\author{
Gislaine Ferro Cordeiro', Maria Gabriela Bernardo da Cunha², Márcia Helena Moreira Menezes³, Maysa Tibério \\ Ubrig-Zancanella ${ }^{4}$, Kátia Nemr ${ }^{5}$
}

\begin{abstract}
RESUMO
Objetivo: Verificar a eficácia dos valores da diferença entre os dois primeiros harmônicos para diferenciar vozes adaptadas de vozes levemente soprosas (B) ou tensas (S), durante a emissão da vogal "é" prolongada. Métodos: Foram avaliadas 30 mulheres com vozes normais e alteradas. Foi gravada a vogal "é" de cada sujeito para posterior extração da intensidade dos dois primeiros harmônicos, por meio do gráfico FFT. Foi feita a subtração da amplitude do primeiro pelo segundo harmônico (H1-H2) e os dados foram tratados estatisticamente. Resultados: A variação entre os valores de H1-H2, entre os sujeitos com grau 0 de soprosidade e tensão e os sujeitos com grau 1, também nos dois parâmetros, foi muito grande, portanto os valores não foram estatisticamente significativos. Entretanto, quando a soprosidade e tensão atingem o grau 2 a variação dos valores aproxima-se das descrições da literatura. Conclusão: A medida estudada não é eficaz para diferenciar vozes adaptadas daquelas levemente soprosas e tensas, em emissões da vogal "é” prolongada.
\end{abstract}

Descritores: Voz/fisiologia; Qualidade da voz; Distúrbios da voz; Acústica da fala

\section{INTRODUÇÃO}

A correlação entre a análise de aspectos fisiológicos, e análises acústica, e perceptivo-auditiva da voz, tem sido muito valorizada por cientistas e clínicos ${ }^{(1,2)}$. Embora a análise acústica seja, em geral, subjetiva ${ }^{(2,3)}$, por meio dela é possível a inferência sobre as características físicas do falante (como sexo ${ }^{(4)}$ e idade $^{(5)}$ ), além da situação glótica ${ }^{(6)}$ e de todo o trato vocal ${ }^{(5)}$.

Um dos recursos da análise acústica, utilizado para associá-

Trabalho realizado no Departamento de Otorrinolaringologia da Faculdade de Medicina da Universidade de São Paulo - USP - São Paulo (SP), Brasil.

(1) Pós-graduanda (Mestrado) em Ciências do Departamento de Otorrinolaringologia da Faculdade de Medicina da Universidade de São Paulo - USP - São Paulo (SP), Brasil.

(2) Mestre, Fonoaudióloga do Hospital das Clínicas da Faculdade de Medicina da Universidade de São Paulo - USP - São Paulo (SP), Brasil.

(3) Pós-graduanda (Doutorado) em Ciências do Departamento de Otorrinolaringologia da Faculdade de Medicina da Universidade de São Paulo - USP - São Paulo (SP), Brasil; Professora do Curso de Fonoaudiologia da Universidade de Guarulhos - UnG - Guarulhos (SP), Brasil.

(4) Pós-graduanda (Mestrado) em Ciências do Departamento de Otorrinolaringologia da Faculdade de Medicina da Universidade de São Paulo - USP - São Paulo (SP), Brasil; Fonoaudióloga do Hospital das Clínicas da Faculdade de Medicina da Universidade de São Paulo - USP - São Paulo (SP), Brasil. (5) Doutora, Professora do Curso de Fonoaudiologia da Faculdade de Medicina da Universidade de São Paulo - USP - São Paulo (SP), Brasil.

Endereço para correspondência: Gislaine Ferro Cordeiro. R. Milton Lodetti, 75, Vl. Leopoldina, São Paulo (SP), Brasil, CEP: 05303-080. E-mail: giscordeiro@usp.br

Recebido em: 3/6/2009; Aceito em: 24/9/2009 la à fisiologia da produção vocal, é a relação H1-H2. Essa medida parece se relacionar diretamente à velocidade das forças aerodinâmicas das pregas vocais ${ }^{(7)}$ e por isso, é bastante utilizada para descrever as características de emissão da voz ${ }^{(8-10)}$.

A amplitude do primeiro harmônico é associada ao grau de excursão lateral das pregas vocais durante a produção da voz, e os harmônicos mais altos à descontinuidade que ocorre com o impacto do contato das pregas vocais ${ }^{(11)}$. Quanto maior o valor do primeiro harmônico, em relação ao segundo, menor é o coeficiente de contato ${ }^{(7,11)} \mathrm{e}$ maior a velocidade de fechamento das pregas vocais ${ }^{(7)}$.

Assim, valores muito altos de $\mathrm{H} 1-\mathrm{H} 2$ podem indicar grande soprosidade e/ou astenia ${ }^{(12)}$, enquanto valores baixos podem indicar tensão/estrangulamento na $\mathrm{voz}^{(13)}$.

Embora a correlação entre os eventos fisiológicos e as medidas referentes aos $\mathrm{H} 1-\mathrm{H} 2$ tenha sido descrita na literatura, esse índice ainda não foi parametrizado à avaliação perceptivo auditiva, considerando as variáveis soprosidade e tensão.

Sendo assim, o objetivo desse estudo é correlacionar e verificar a eficácia dos valores da diferença de amplitude dos dois primeiros harmônicos com a avaliação perceptivo-auditiva de vozes normais e levemente soprosas e tensas, durante a emissão da vogal "é" sustentada.

\section{MÉTODOS}

Foram selecionados 30 indivíduos adultos voluntários, do 
sexo feminino, com idades variando entre 22 e 72 anos, provenientes do ambulatório da Divisão de Otorrinolaringologia do Hospital das Clínicas da Faculdade de Medicina da Universidade de São Paulo (HC-FMUSP), com e sem queixas vocais.

Todos os indivíduos participantes responderam e assinaram o Termo de Consentimento Livre e Esclarecido. O projeto de pesquisa foi aprovado pela Comissão de Ética para Análise de Projetos de Pesquisa do HC-FMUSP, sob número 1017/08, em 06 de fevereiro de 2009.

Os sujeitos foram divididos em dois grupos: o grupo 1 foi composto por 17 mulheres sem disfonia e o grupo 2 por 13 mulheres disfônicas. Todos os indivíduos foram submetidos à triagem perceptivo-auditiva, por meio da escala $\operatorname{GRBAS}^{(14,15)}$ e videoestrobolaringoscopia.

Os sujeitos posicionaram-se sentados em uma cadeira com apoio de cabeça, dentro de uma cabine acústica. Um microfone headset unidirecional da marca Plantronics $\AA$, colocado a uma distância de três centímetros da boca do indivíduo capturou a voz, gravada e armazenada diretamente num computador Pentium II. Os sinais foram digitalizados e editados no software Sound Forge 6.0.

Foi pedido aos indivíduos que emitissem vogal "é" prolongada, de maneira natural e confortável, em intensidade e altura habituais, sem tentar impostar a voz.

As vozes foram avaliadas no programa de análise acústica Praat versão 4.6.06 por meio de análise do espectro, extraído do espectrograma de banda estreita, em que o cálculo para a visualização dos harmônicos foi obtido pelo Fast Fourier Transform (FFT - Transformada Rápida de Fourier). Foram selecionados três momentos do meio da emissão de cada sujeito e escolhido o de melhor representatividade vocal. Os parâmetros do espectrograma de curto termo foram "window length $=0,05$ seg" e "dynamic range $=40 \mathrm{~dB}$ ".

Foram selecionados os picos dos dois primeiros harmônicos, extraídas as amplitudes respectivas de cada um, para então subtrair a amplitude do primeiro à do segundo harmônico. As análises foram feitas de modo que o avaliador não soubesse o resultado da avaliação videoestroboscópica e perceptivo-auditiva.

Após as análises, os valores foram tabulados, juntamente com os resultados de soprosidade (B) e tensão (S) da triagem perceptivo-auditiva. Os dados foram tratados estatisticamente por meio do teste t-independente com o nível de significância de 5\%.

\section{RESULTADOS}

As médias de H1-H2 das vozes normais, soprosas e tensas, bem como seus desvios padrão foram demonstrados nas Tabelas 1,2 e 3.

As Figuras 1, 2, 3 e 4 mostram os espectros de vozes normais, soprosidade leve, soprosidade moderada, tensão leve, respectivamente.

Tabela 1. Comparação de média e desvio-padrão da diferença H1-H2 em vozes sem e com soprosidade

\begin{tabular}{lccc}
\hline Medidas & \multicolumn{2}{c}{$\mathrm{B}$ (soprosidade) } & Valor de $\mathrm{p}$ \\
\cline { 2 - 3 } descritivas & Grau 0 & $\begin{array}{r}\text { Grau 1 ou } \\
\text { Grau 2 }\end{array}$ & \\
\hline Média & 1,35 & 3,38 & \\
DP & 6,08 & 6,95 & 0,401 \\
$\mathrm{~N}$ & 17 & 13 & \\
\hline
\end{tabular}

Teste $\mathrm{t}$

Legenda: $\mathrm{DP}=$ desvio-padrão

Tabela 2. Comparação de média e desvio-padrão da diferença $\mathrm{H} 1-\mathrm{H} 2$ em vozes sem e com soprosidade de graus leve e moderado

\begin{tabular}{lccc}
\hline Medidas & \multicolumn{3}{c}{ B (soprosidade) } \\
\cline { 2 - 4 } descritivas & Grau 0 & Grau 1 & Grau 2 \\
\hline Média & 1,35 & 2,45 & 8,50 \\
DP & 6,08 & 7,19 & 0,71 \\
N & 17 & 11 & 2 \\
\hline
\end{tabular}

Legenda: DP = desvio-padrão

Tabela 3. Comparação de média e desvio padrão da diferença entre $\mathrm{H} 1-\mathrm{H} 2$ em vozes sem e com tensão

\begin{tabular}{lccc}
\hline Medidas & \multicolumn{2}{c}{$\mathrm{S}$ (tensão) } & \multirow{2}{*}{ Valor de $\mathrm{p}$} \\
\cline { 2 - 3 } descritivas & Grau 0 & Grau 1 & \\
\hline Média & 1,50 & 2,88 & \\
DP & 6,71 & 6,33 & 0,569 \\
$\mathrm{~N}$ & 14 & 16 & \\
\hline
\end{tabular}

Teste $\mathrm{t}$

Legenda: DP = desvio-padrão

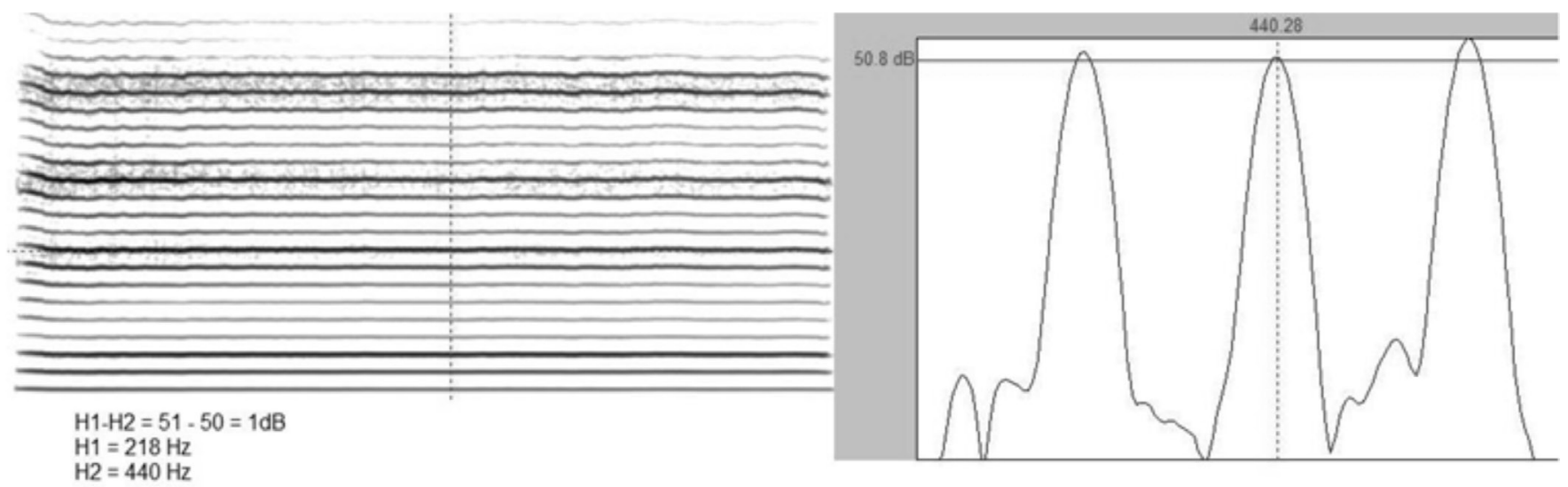

Figura 1. Espectrograma e espectro da vogal "é" sustentada com grau de tensão e soprosidade igual a 0. Diferença entre os harmônicos é igual a $1 \mathrm{~dB}$. O primeiro harmônico tem a frequência de $218 \mathrm{~Hz}$ e o segundo de $440 \mathrm{~Hz}$. 

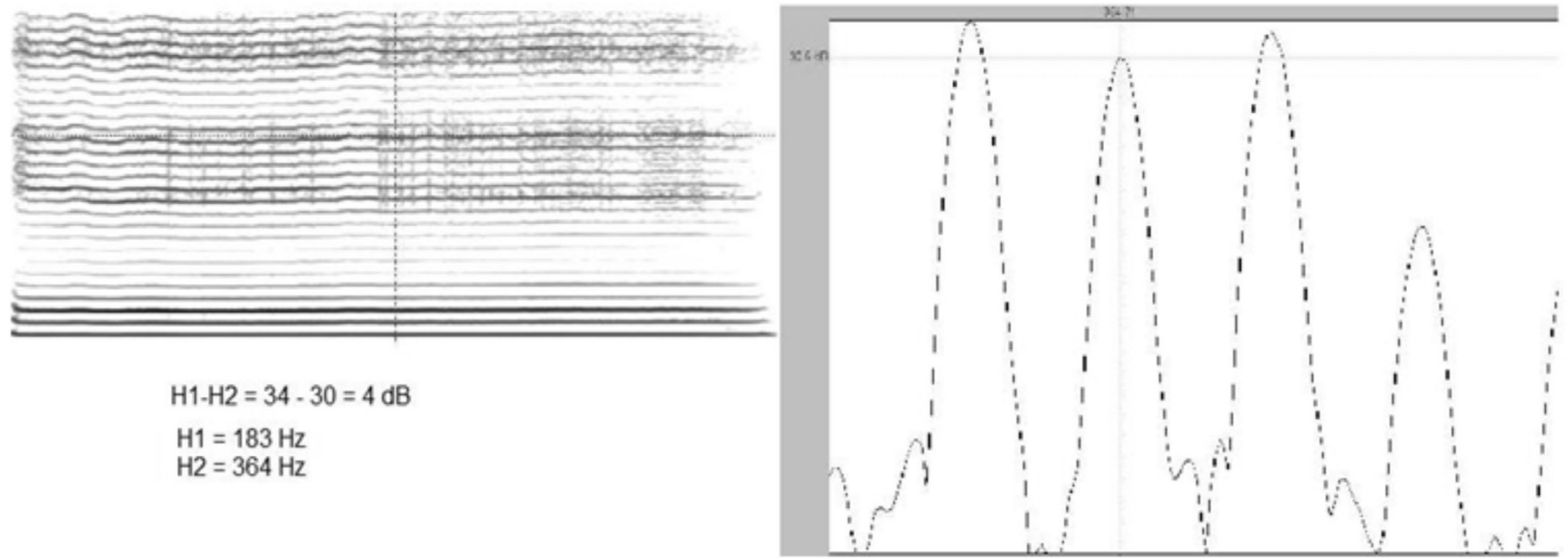

Figura 2. Espectrograma e espectro de vogal "é" sustentada com grau de soprosidade igual a 1. Diferença entre os harmônicos é igual a 4 dB. O primeiro harmônico tem a frequência de $183 \mathrm{~Hz}$ e o segundo de $364 \mathrm{~Hz}$.

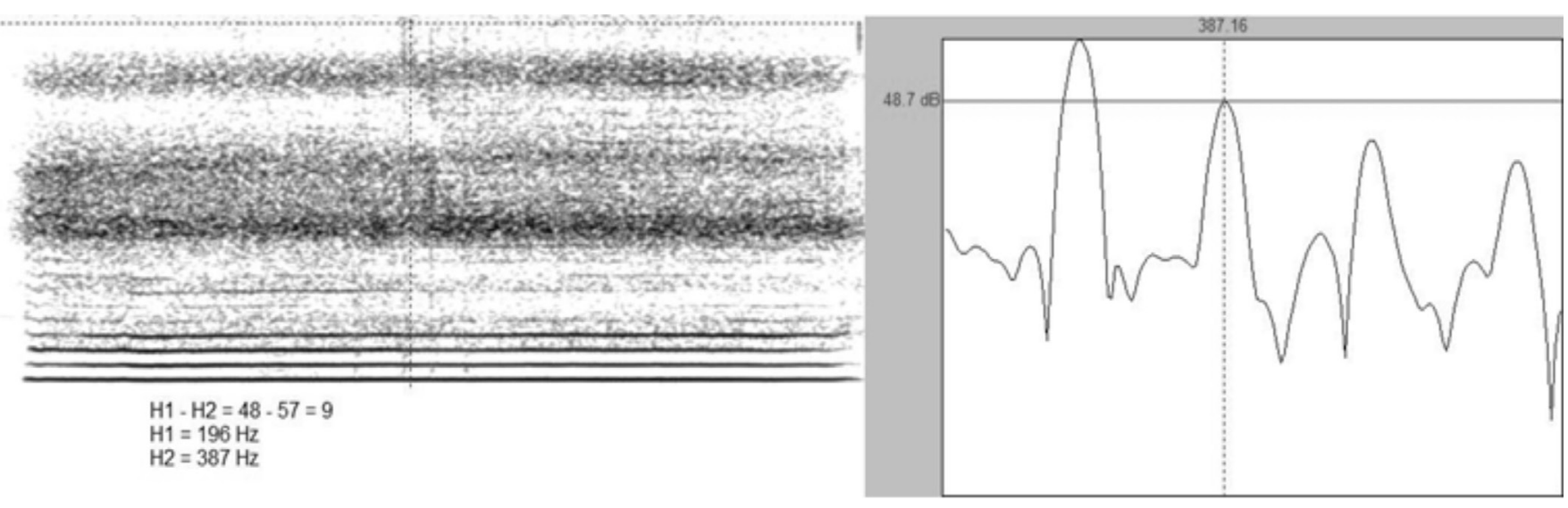

Figura 3. Espectrograma e espectro de vogal "é" sustentada com grau de soprosidade igual a 2. Diferença entre os harmônicos é igual a 9 dB. O primeiro harmônico tem a frequência de $196 \mathrm{~Hz}$ e o segundo de $387 \mathrm{~Hz}$.
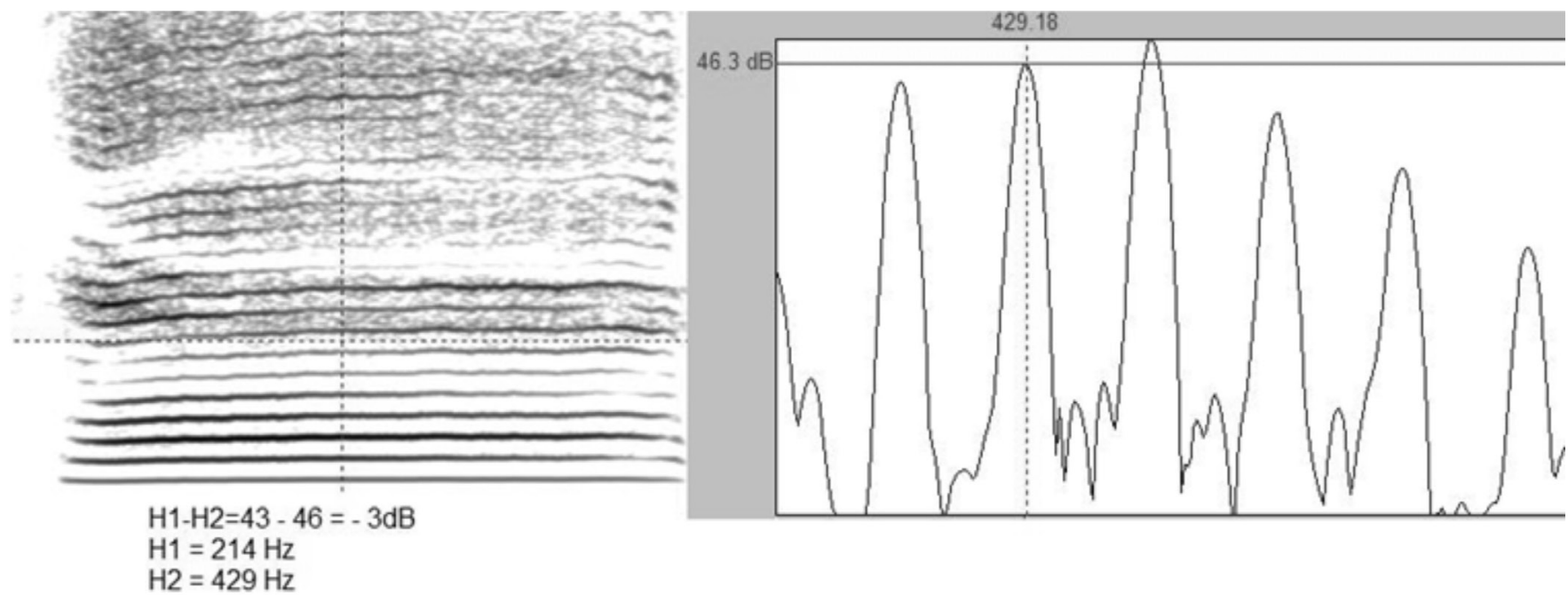

Figura 4. Espectrograma e espectro de vogal "é" sustentada com grau de tensão igual a 1. Diferença entre os harmônicos é igual a -3 dB. $O$ primeiro harmônico tem a frequência de $214 \mathrm{~Hz}$ e o segundo de $429 \mathrm{~Hz}$

Não foram encontrados valores estatisticamente significantes no que diz respeito a $\mathrm{H} 1-\mathrm{H} 2$, das vozes levemente alteradas (quanto à soprosidade e tensão) em relação às vozes adaptadas, pois o desvio padrão foi muito grande para os dois tipos de vozes. Entretanto, quando o grau de soprosi- dade chega a 2 , os valores tendem a ser significantes, pois a média da diferença entre as intensidades dos dois primeiros harmônicos alcança valor quatro vezes maior do que a média de soprosidade de grau 1. Além disso, o desvio padrão nesses casos é muito pequeno. 


\section{DISCUSSÃO}

Embora a variação de idade entre os sujeitos seja grande, acreditamos que essa variável não tenha tido interferência em nosso estudo, pois o correlato fisiológico da diferença entre as amplitudes dos dois primeiros harmônicos é o grau de excursão lateral das pregas vocais durante a produção da voz ${ }^{(11)}$, o que interferiria na percepção de soprosidade ${ }^{(12)}$ e tensão ${ }^{(13)}$, independentemente da idade do falante.

Para a avaliação perceptivo-auditiva, foi utilizada a escala GRBAS, criada pela Sociedade Japonesa de Logopedia e amplamente divulgada por Hirano ${ }^{(14,15)}$. A GRBAS é um instrumento de avaliação perceptivo-auditivo amplamente divulgado e utilizado por clínicos e cientistas do mundo. Ela considera os parâmetros vocais de alterações glóticas, sendo $\mathrm{G}$ (grade) grau geral da disfonia, $\mathrm{R}$ (rough) rugosidade, B (breathy) soprosidade, A (astheny) astenia, S (strain) soprosidade, nos graus 0 (normal), 1 (leve), 2 (moderado), 3 (intenso). Para nosso estudo, utilizamos os parâmetros B (soprosidade) e S (tensão) por serem os correlatos de hipo e hiperadução glótica ${ }^{(16)}$, relacionados à diferença dos dois primeiros harmônicos ${ }^{(7,11,12)}$. A videolaringoestroboscopia foi realizada em todos os sujeitos com o intuito de corroborar por meio da imagem laríngea a presença ou não de alteração, confirmando os achados perceptivo-auditivos, neste caso para a definição dos dois grupos.

A vogal "é" foi escolhida por ser considerada uma das vogais mais próximas do “æ”, em que há maior amplitude da cavidade oral e da faringe ${ }^{(17)}$ e também por ser comumente utilizada no exame otorrinolaringológico, fonoaudiológico e em pesquisas ${ }^{(18-20)}$. No entanto, é importante que se faça validação dessa medida em todas as vogais da língua portuguesa, pois em algumas vogais o primeiro formante pode modificar a medida do segundo harmônico.

O formante da vogal "é" em mulheres do Estado de São Paulo, está por volta de $640 \mathrm{~Hz}^{(21)}$, em nossa pesquisa, o segundo harmônico estava por volta de $400 \mathrm{~Hz}$. Embora o valor esteja próximo da média do primeiro formante, todos os sujeitos da pesquisa tiveram as mesmas condições de interferência do trato vocal, não sendo essa uma diferença significativa na conclusão final desse estudo. É interessante que se pesquise o emprego da vogal "a" nesse tipo de estudo.

De acordo com a literatura, quanto maior a diferença entre $\mathrm{H} 1-\mathrm{H} 2$, menor o contato das pregas vocais durante a fonação e vice-versa ${ }^{(7,11-13)}$. Por exemplo, no estilo de canto ópera, os valores de $\mathrm{H} 1-\mathrm{H} 2$, são maiores do que no estilo musical theater ${ }^{(22)}$.

A literatura relata que em pacientes com paralisia vocal, os valores de amplitude do primeiro harmônico são maiores do que os do segundo ${ }^{(11)}$. O contrário também é verdadeiro, já que esses valores tornam-se negativos em pacientes com disfonia espasmódica de adução ${ }^{(12)}$.

Entretanto, em nossos achados, quando os valores da diferença H1-H2 das vozes levemente soprosas ou tensas são comparados às vozes normais, não são significativos estatisti- camente $^{(6,23)}$. De acordo com pesquisas realizadas em sujeitos normais, asmáticos e com movimento paradoxal de pregas vocais $^{(12)}$ e em alterações estruturais leves como nódulos de pregas vocais ${ }^{(6)}$, os valores dessas medidas não são estatisticamente significativos.

Assim, nos trabalhos em que as diferenças de tensão e soprosidade são mais importantes, essa medida parece ser mais significativa do que naqueles em que a alteração é mais sutil. Dessa maneira, acreditamos que o ouvido humano ainda é mais sensível às pequenas alterações de voz $^{(24,25)}$, quando comparado a essas medidas de amplitude, que podem ser eficazes para grandes alterações do coeficiente de abertura e fechamento das pregas vocais.

Essa hipótese foi levantada quando dois de nossos sujeitos apresentaram grau moderado de soprosidade e os valores de $\mathrm{H} 1$ em relação a $\mathrm{H} 2$, tenderam a ser significantemente mais altos. Embora esses sujeitos não tenham apresentado grau leve de soprosidade, e representam um número de amostragem muito pequeno, não foram excluídos do estudo por terem apresentado resultados diferenciados em relação aos sujeitos de grau leve. Assim, sugerimos que novos estudos sejam realizados com o intuito de parametrizar e verificar a eficácia da diferença de $\mathrm{H} 1-\mathrm{H} 2$ em vozes com grau moderado e intenso de soprosidade e tensão.

A diferença entre os dois primeiros harmônicos também foi descrita como favoráveis para a diferenciação de registros vocais intra-sujeitos ${ }^{(13)}$. Na voz modal, onde o fechamento glótico é maior ${ }^{26)}$, o valor do segundo harmônico tende a ser mais alto do que no falsete ${ }^{(13)}$, onde o fechamento glótico é menor $^{(26)}$.

Dessa maneira acreditamos que essa medida possa ser eficaz para verificar variações intra-sujeitos, mesmo diante de pequenas diferenças de voz. Sugerimos então, que sejam realizados estudos com diversos graus de disfonias, antes e depois dos respectivos tratamentos.

Além disso, essa pesquisa foi realizada em uma única vogal, que não necessariamente é representativa da emissão de fala espontânea ou encadeada. Consideramos, então esse estudo como piloto para outros, que realizem a extração dessa medida durante essas tarefas fonatórias.

Acreditamos, também, que a diferença de $\mathrm{H} 1-\mathrm{H} 2$ possa ser uma medida importante no diagnóstico e acompanhamento do tratamento das disfonias, mas que deve ser utilizada como complemento nas avaliações de voz, já que o ouvido humano parece ser ainda mais sensível diante das discretas diferenças vocais apresentadas pelo paciente ${ }^{(24,25)}$.

\section{CONCLUSÃO}

A diferença da medida de amplitude dos dois primeiros harmônicos (H1-H2) não é eficaz para detectar alterações vocais leves de soprosidade e tensão na emissão da vogal "é" sustentada. 


\begin{abstract}
Purpose: To verify the efficacy of the values of the difference between the first two harmonics to distinguish adapted voices from slightly breathy (B) or tense (S), during the emission of the long vowel /él. Methods: Thirty women with normal and altered voices were evaluated. Each subject's vowel "é" was recorded in order to extract the intensity of the first two harmonics, using the FFT graph. The range of the second harmonic was subtracted from the range of the first, and data were treated statistically. Results: The variation between the values of $\mathrm{H} 1-\mathrm{H} 2$ between subjects rated 0 breathiness and tension and subjects rated 1 , also in both parameters, was very high, therefore the values were not statistically significant. However, when the degrees of breathiness and tension get to 2 , the variation values become more compatible with descriptions in literature. Conclusion: The measure studied is not efficient to distinguish adapted voices from those slightly breathy and tense, during the emission of the long vowel /é/.
\end{abstract}

Keywords: Voice/physiology; Voice quality; Voice disorders; Speech acoustics

\section{REFERÊNCIAS}

1. Laver J, Mackenzie-Beck J. Vocal Profile Analysis Scheme-VPAS: a user's manual. Edinburgh: Queen Margaret Universit; 2007.

2. Camargo Z, Pinho SRM. Introdução à análise acústica da voz. In: Pinho SEM, organizador. Tópicos em voz. Rio de Janeiro: Guanabara-Koogan; 2001. p. 19-44.

3. Behlau MS. Voz: O livro do especialista. Rio de Janeiro: Revinter; 2006. Avaliação de voz; p. 85-245.

4. Holmberg EB, Hillman RE, Perkell JS. Glottal airflow and transglottal air pressure measurements for male and female speakers in soft, normal, and loud voice. J Acoust Soc Am. 1988;84(2):511-29.

5. Linville SE, Rens J. Vocal tract resonance analysis of aging voice using long-term average spectra. J Voice. 2001;15(3):323-30.

6. Holmberg EB, Doyle P, Perkell JS, Hammarberg B, Hillman RE. Aerodynamic and acoustic voice measurements of patients with vocal nodules: variation in baseline and changes across voice therapy. J Voice. 2003;17(3):269-82.

7. Hammarberg B, Fritzell B, Gauffin J, Sundberg J, Wedin L. Perceptual and acoustics correlates of abnormal voice qualities. Acta Otolaryngol. 1980;90(5-6):441-51.

8. Klatt DH, Klatt LC. Analysis, synthesis, and perception of voice quality variations among females and male talkers. J Acoust Soc Am. 1990;87(2):820-57.

9. Hanson HM. Glottal characteristics of female speakers: acoustic correlates. J Acoust Soc Am. 1997;101(1):466-81.

10. Hanson HM, Chuang ES. Glottal characteristics of male speakers: acoustic correlates and comparison with female data. J Acoust Soc Am. 1999;106(2):1064-77.

11. Hart DA, Hans S, Vaissière J, Brasnu DA. Objective acoustic and aerodynamic measures of breathiness in paralytic dysphonia. Eur Arch Otorynolaringol. 2003;260(4):175-82.

12. Cannito MP, Buder EH, Chorna LB. Spectral amplitude measures of adductor spasmodic dysphonic speech. J Voice. 2005;19(3):391-410.

13. Salomão GL, Sundberg J. Relation between perceived voice register and flow glottogram parameters in males. J Acoust Soc Am. 2008;124(1):546-51

14. Isshiki, N. Olamura H, Tanabe M, Morimoto M. Differential diagnosis of hoarseness. Folia Phoniatr (Basel). 1969;21(1):9-19.
15. Hirano N. Clinical examination of voice. Viena: Springer Verlag; 1981.

16. Pinho SMR, Pontes PAL. Músculos Intrínsecos da laringe e dinâmica vocal. Rio de Janeiro: Revinter; 2008.

17. Story BH, Titze IR, Hoffman EA. Vocal tract area functions for an adult female speaker based on volumetric imaging. J Acoust Soc Am. 1998;104(1):471-87

18. Gama ACC, Behlau MS. Estudo da constência de medidas acústicas de vogais prolongadas e consecutivas em mulheres sem queixa de voz e em mulheres com disfonia. Rev Soc Bras Fonoaudiol. 2009;14(1):8-14

19. Ciocchi PE, Andrade, CRF. Protocolo de cooperação fonoaudiológica para avaliação nasofibrolaringoscópica da mobilidade laríngea em doenças da tireóide (PAN). Pró-fono. 2009;21(1):31-8.

20. Cunha MGB. Avaliação de pacientes portadores de obesidade mórbida candidatos à cirurgia bariátrica [dissertação]. São Paulo: Faculdade de Medicina, Universidade de São Paulo; 2008.

21. Teles VC, Rosinha ACU. Análise acústica dos formantes e das medidas de perturbação do sinal sonoro em mulheres sem queixas vocais, não fumantes e não estilista. Arq Int Otorrinolaringol. 2008;12(4):523-30.

22. Björkner E. Musical theater and opera singing - why so different? A study of subglottal pressure, voice source, and formant frequency characteristics. J Voice. 2008;22(5):533-40

23. Cukier S. Qualidade vocal em indivíduos asmáticos com e sem disfunção paradoxal de pregas vocais: correlatos perceptivos auditivos, acústicos e fisiológicos [dissertação]. São Paulo: Pontifícia Universidade Católica; 2006.

24. Pereira Jotz G, Cervantes O, Abrahão M, Parente Settanni FA, Carrara de Angelis E. Noise-to-harmonics ratio as an acoustic measure of voice disorders in boys. J Voice. 2002;16(1):28-31.

25. Nemr K, Amar A, Abrahão M, Leite GCA, Köhle J, Santos AO, et al. Análise comparativa entre avaliação fonoaudiológica perceptivoauditiva, análise acústica e laringoscopias indiretas para avaliação vocal em população com queixa vocal. Rev Bras Otorrinolaringol. 2005;71(1):13-7

26. Hirano M, Vennard W, Ohala J. Regulation of register, pitch and intensity of voice: An electromyographic investigation of intrinsic laryngeal muscles. Folia Phoniatr (Basel). 1970;22(1):1-20. 